\title{
Clinical efficacy of laser therapy in the prevention of retinal detachment in patients with acquired immunodeficiency syndrome and cytomegalovirus retinitis
}

\author{
Lian-Yong Xie ${ }^{1 \#}$, Xiao-Na Li ${ }^{1 \#}$, Chao Chen ${ }^{1}$, Wen-Jun Kong ${ }^{1}$, Tai-Yi Jiang ${ }^{2}$, Kui-Fang Du ${ }^{1}$, \\ Hong-Wei Dong ${ }^{1}$, Chun-Gang Guo ${ }^{1}$, Wen-Bin Wei ${ }^{3}$
}

${ }^{1}$ Department of Ophthalmology, Beijing YouAn Hospital, Capital Medical University, Beijing, China; ${ }^{2}$ Department of Infectious Diseases, Beijing YouAn Hospital, Capital Medical University, Beijing, China; ${ }^{3}$ Beijing Tongren Eye Center, Beijing Key Laboratory of Intraocular Tumor Diagnosis and Treatment, Beijing Ophthalmology \& Visual Sciences Key Lab, Medical Artificial Intelligence Research and Verification Key Laboratory of the Ministry of Industry and Information Technology, Beijing Tongren Hospital, Capital Medical University, Beijing, China

\#These authors contributed equally to this work.

Correspondence to: Wen-Bin Wei. Beijing Tongren Eye Center, Beijing Key Laboratory of Intraocular Tumor Diagnosis and Treatment, Beijing Ophthalmology \& Visual Sciences Key Lab, Medical Artificial Intelligence Research and Verification Key Laboratory of the Ministry of Industry and Information Technology, Beijing Tongren Hospital, Capital Medical University, No. 1 Dongjiaomin Lane, Dongcheng District, Beijing 100730, China. Email: weiwb1023@163.com.

Background: The aim of the present study was to evaluate the clinical efficacy of laser therapy in the prevention of retinal detachment in patients with acquired immunodeficiency syndrome (AIDS) and cytomegalovirus retinitis (CMVR).

Methods: A total of 96 eyes from 80 patients with AIDS and CMVR who received anticytomegalovirus (anti-CMV) treatment in the ophthalmology and infection centers of Beijing YouAn Hospital, between June 2016 and August 2018 were retrospectively investigated. The patients were randomly divided into a nonlaser group (50 eyes from 43 patients), who were treated with anti-CMV therapy, and a laser group (46 eyes from 37 patients), who were treated with a fundus laser method to close the retinopathy area after commencing the maintenance stage of anti-CMV treatment. Both groups were followed up for 24 months. The safety of laser therapy was observed, and the efficacy of the therapy was determined by evaluating the incidence of retinal detachment.

Results: The percentage of retinal detachment in the nonlaser group was $24 \%$ compared with $6.5 \%$ in the laser group $(\mathrm{P}=0.018)$. There was no significant difference between the two groups in the number of CD4 ${ }^{+}$ $T$ cells, the load of human immunodeficiency virus, or the time between the detachment and the end of the induction period. After laser therapy, 39.13\% of patients exhibited keratic precipitates (KP), 30.43\% had anterior chamber flare $( \pm), 50 \%$ had anterior chamber flare (+), and $19.57 \%$ had anterior chamber flare (++). Intraocular pressure (IOP) increased in 3 eyes within 2 weeks of laser therapy. The retinal pigment reaction was not obvious in 8 eyes.

Conclusions: The use of laser therapy in the main maintenance period of anti-CMV treatment can effectively reduce the incidence of retinal detachment in patients with AIDS and CMVR, and the therapy is safe and reliable.

Keywords: Cytomegalovirus retinitis (CMVR); laser therapy; retinal detachment; therapeutic efficacy

Submitted Aug 24, 2020. Accepted for publication Feb 04, 2021.

doi: 10.21037/qims-20-990

View this article at: http://dx.doi.org/10.21037/qims-20-990 


\section{Introduction}

Cytomegalovirus retinitis (CMVR) is the main cause of vision loss in patients with acquired immunodeficiency syndrome (AIDS) (1-3). It directly damages the retina, the macular area, and the optic papilla, and can result in vision loss due to complications such as immune reconstitution uveitis, macular edema, or cataracts (4). However, the most serious complication is retinal detachment, which generally has a devastating effect on the patient's vision $(4,5)$. Research has shown that the incidence of retinal detachment is $50 \% 1$ year after the diagnosis of CMVR (6). Fullthickness retinal necrosis and retinal detachment caused by CMVR require complicated operations such as retinotomy, which is a necessary procedure during vitrectomy surgery. Retinal reattachment is affected by many factors, and the therapeutic effect is generally unsatisfactory. In recent years, it has been reported that fundus laser therapy can prevent retinal detachment in the later stages of CMVR and results in less damage and fewer complications (7-9). In our hospital, when possible, we use fundus laser to block the necrotic area of the lesion to prevent retinal detachment in patients with stable CMVR. This report details our findings.

\section{Methods}

\section{General data}

This study was approved by the Capital Medical University Institutional Review Board of Beijing YouAn Hospital (LL2018-150-K) and adhered to the tenets of the Declaration of Helsinki. Written informed consent was obtained from all participants. A total of 96 eyes from 80 patients with AIDS and CMVR who were diagnosed and treated in our hospital between June 2016 and August 2018 were retrospectively investigated. AIDS was diagnosed by the Department of Infection in our hospital, while CMVR was diagnosed by two experienced doctors in our department according to the history of HIV infection, the clinical manifestations, the typical fundus appearance of "cottage cheese and ketchup", the positive CMV-DNA in the aqueous humor, and the number of serum $\mathrm{CD}^{+} \mathrm{T}$ cells. The eyes in question were treated with anticytomegalovirus (anti-CMV) therapy in our hospital, and patients were aged between 20 and 56 years. The average visual acuity (counting fingers/ anterior) was -1.0 .

We divided the patients into nonlaser and laser groups according to whether they received laser treatment. The nonlaser group patients were treated with anti-CMV 50 therapy alone. Meanwhile, the laser group received fundus 51 laser therapy to close the lesion area when the induction 52 period of the anti-CMV treatment had ended and the 53 disease was stable. All patients were followed up for 54 24 months to observe the incidence of complications and 55 retinal detachment.

\section{Exclusion criteria}

The exclusion criteria were as follows: (I) retinal detachments occurring before the end of the induction period of the anti-CMV therapy; (II) retinal detachments occurring after relapse of CMVR in the maintenance period involving anti-CMV therapy; (III) patients with CMVR caused by other factors (e.g., CMVR after transplantation); (IV) patients with AIDS, CMVR, or lymphoma who required immunosuppressants; (V) patients with other serious ocular diseases, such as diabetic retinopathy or high myopia; and (VI) patients with a pre-existing anterior chamber reaction.

\section{Anti-CMV therapy}

A $5-\mathrm{mg} / \mathrm{kg} /$ day dosage of ganciclovir or a $60-90-\mathrm{mg} /$ day dosage of foscarnet sodium was administered intravenously during the induction period. Furthermore, $3 \mathrm{mg} /$ time of ganciclovir or $2.4 \mathrm{mg} /$ time of foscarnet sodium was injected into the vitreous body where appropriate, twice a week for 3 weeks. During the maintenance period, ganciclovir was given orally at a dosage of $3 \mathrm{~g} /$ day until there were no active lesions in the fundus for at least 6 months, the number of $\mathrm{CD}^{+} \mathrm{T}$ cells reached more than $150 / \mu \mathrm{L}$, and the HIV viral load was reduced to undetectable levels. Vitrectomy was performed in the case of retinal detachment.

\section{Laser therapy}

The patients with CMVR were treated with a systemic and local injection of ganciclovir for 3 weeks. After commencing the maintenance period with stable fundus lesions, laser therapy was performed selectively. Here, a multi-wavelength laser photocoagulation instrument (MC500, NIDEK, Japan) and 165-degree panretinoscopy were adopted. Obucaine was selected as the surface anesthesia and ofloxacin eye ointment as the coupling agent. The parameter settings were as follows: power $=100-400 \mathrm{mw}$; spot diameter $=50-400 \mu \mathrm{m}$; and blasting time $=0.1-0.2 \mathrm{~s}$. 

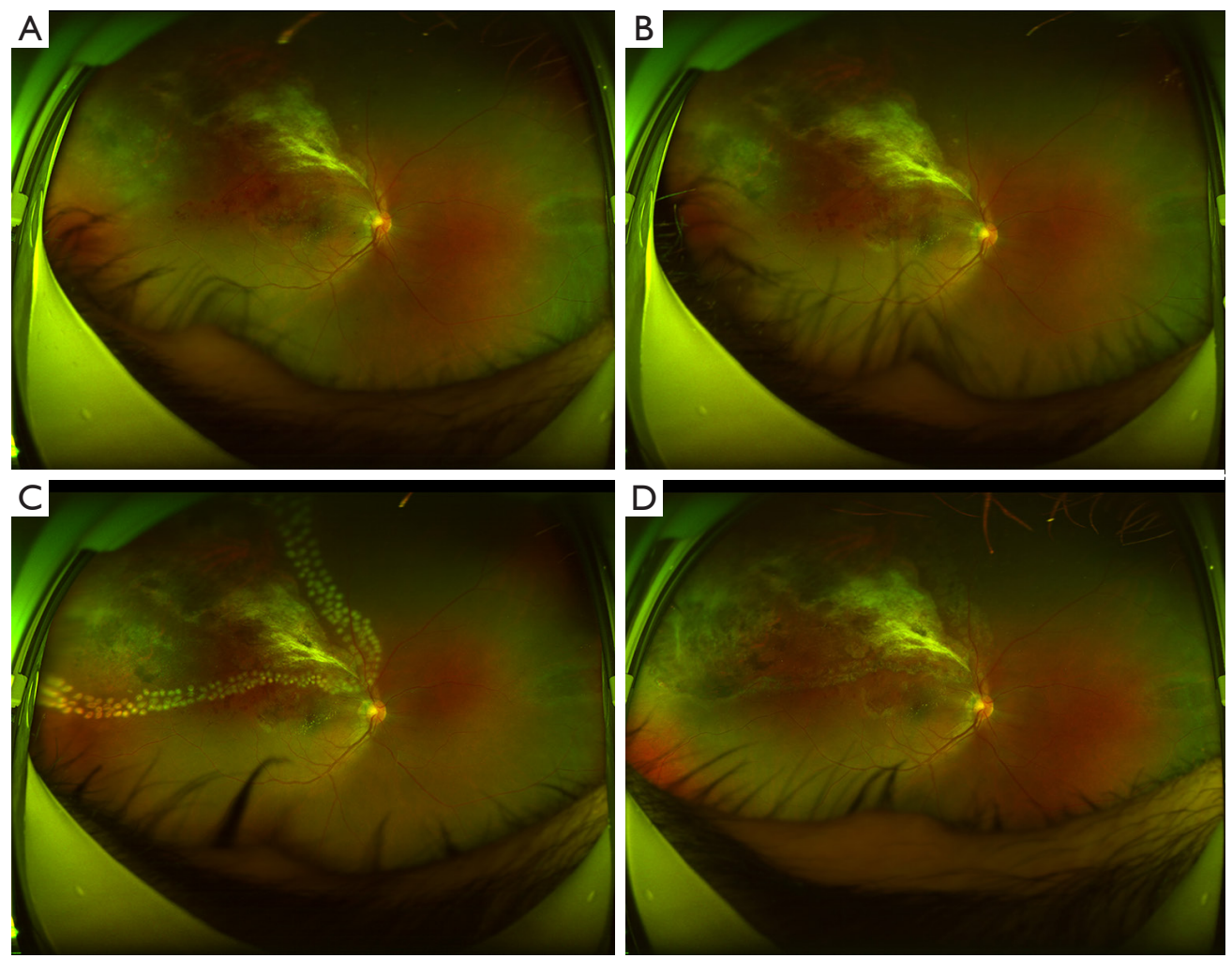

Figure 1 Typical case 1. Laser treatment when CMVR occurs in a quadrant of the retina. (A) Before anti-CMV induction therapy; (B) after anti-CMV induction therapy; (C) after laser therapy; (D) at follow-up. CMVR, cytomegalovirus retinitis; anti-CMV, anticytomegalovirus.

Additionally, a 532-nm green wavelength laser was used in the transparent refractive stroma state, a $577-\mathrm{nm}$ yellow wavelength laser was used when the refractive stroma was turbid, and a $300-400 \mu \mathrm{m}$ spot diameter was selected in the peripheral region. Meanwhile, yellow light was selected in the upper and lower vascular arch of the macular area, and a $50-100 \mu \mathrm{m}$ spot diameter was used. The exposure time was $0.05-0.1 \mathrm{~s}$. The laser parameters were adjusted according to the response of the retinal spot, and 2-4 rows of encircling and intercepting laser photocoagulation were carried out $300-600 \mu \mathrm{m}$ behind the normal retina at the junction of the necrotic retinal focus and the normal retina. The light spot reaction reached level II-III, and the inner and outer circles were mutually interlaced. Two weeks after the operation, "dyke-like" photocoagulation spots and pigmentation appeared on the edge of the necrotic retinal focus, while the number of laser points was within 500 points/time. The case details were recorded, and photos were taken using a wide-angle camera (OPTOS PLC) before and after the laser therapy.

Levofloxacin eye drops were administered 1-3 days before the laser therapy 4 times a day. For the laser room preparation, 165-degree panretinoscopy was used for the patients with AIDS, while a face baffle was installed for the laser slit lamp. The operator wore a mask and gloves to avoid skin damage to the hands. Levofloxacin eye ointment was used as the coupling agent. Following completion of the laser therapy, the mandibular bracket of the laser slit lamp was cleaned with chlorine disinfectant, and the laser room was irradiated with ultraviolet light for 1 hour. The panretinoscope was wiped with a $75 \%$ alcohol solution, soaked in glutaraldehyde for 20 minutes, and then washed and dried with water for further use. The patients were treated with topical pranoprofen, tobramycin, and dexamethasone eye drops for 3 days and were re-examined on days 1 and 3, and weeks 1 and 2 after the operation. Two typical cases are shown in Figures 1 and 2, respectively.

\section{Examinations}

Visual acuity (logarithmic visual acuity chart), intraocular pressure (IOP), slit lamp, and indirect ophthalmoscopy 

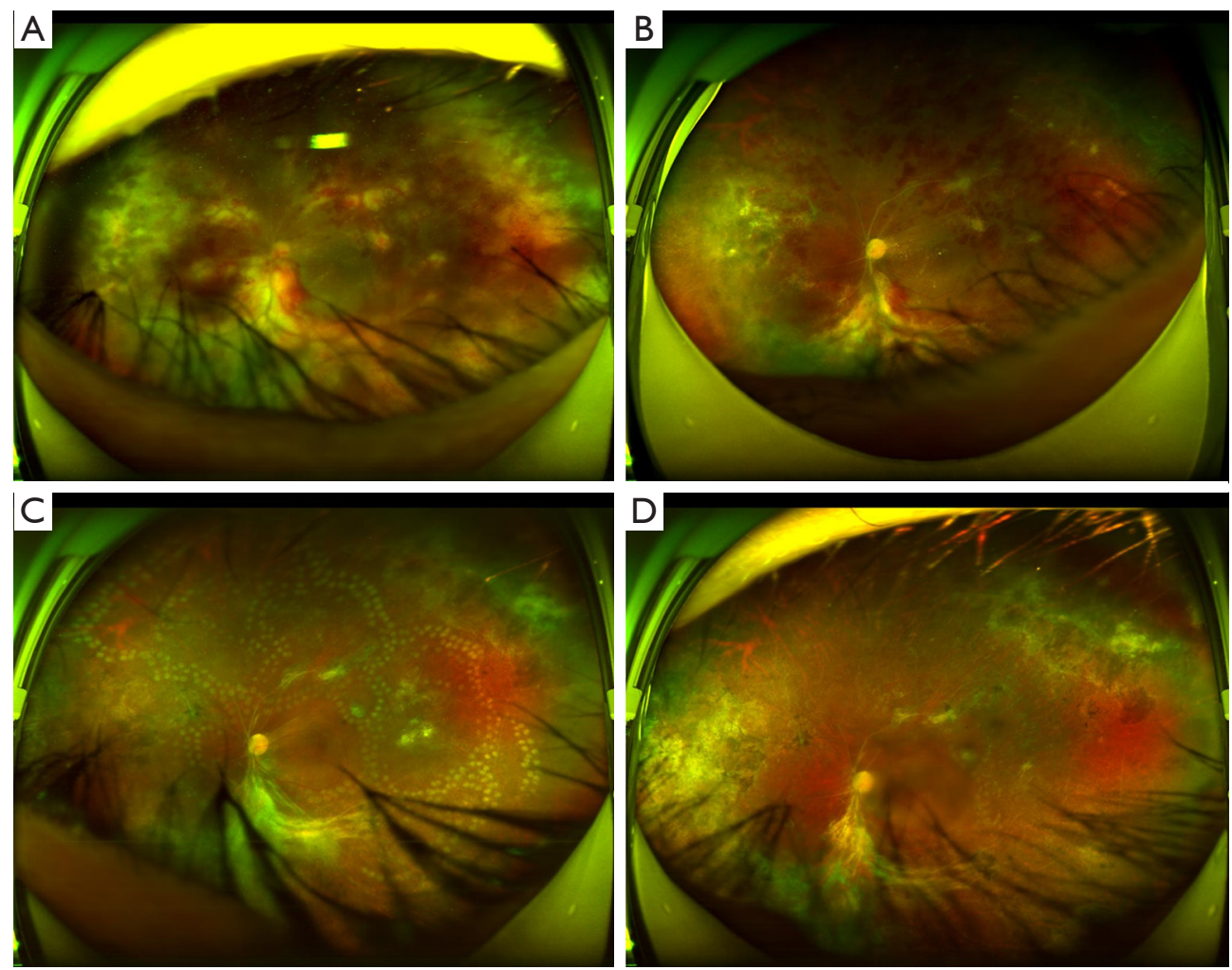

Figure 2 Typical case 2. Laser treatment when CMVR is present in all four quadrants of the retina. For such patients with severe disease, laser treatment was still carried out and certain visual function was maintained for patients. (A) Before anti-CMV induction therapy; (B) after anti-CMV induction therapy; (C) after laser therapy; (D) 1 month after laser therapy. CMVR, cytomegalovirus retinitis; anti-CMV, anticytomegalovirus.

examinations were performed for all eyes, with the results recorded in detail. Fundus fluorescein angiography (FFA) and choroidal angiography (chorography) were also performed, and photos were taken using the wideangle camera. The patients were followed up regularly for 24 months.

\section{Statistical analysis}

SPSS v.22 (IBM, Chicago, USA) software (IBM Corp., USA) was used for the statistical analysis. Data that conformed to a normal distribution are expressed as means \pm standard deviations, and between-group comparisons were conducted using $t$-tests or analysis of variance (ANOVA). Data with a nonnormal distribution are expressed as the median (minimum-maximum), and comparisons were completed using the rank-sum test. Frequency data are described as number of cases (percentage). A chi-square test was used to complete between-group comparisons where appropriate, while Fisher's exact test was used when the chisquare test conditions were not met. A P value $<0.05$ was considered to be statistically significant.

\section{Results}

\section{Re-examination on day 1 after laser therapy}

In the laser group, there were 18 eyes (39.13\%) with keratic precipitates (KP), $14(30.43 \%)$ with anterior chamber flare $( \pm), 23(50 \%)$ with anterior chamber flare (+), and $9(19.57 \%)$ with anterior chamber flare $(++)$. The anterior chamber reaction had disappeared by the time of re-examination 1 week after the operation. The IOP had increased in 3 eyes within 2 weeks of the operation, with a fluctuation of 21-28 $\mathrm{mmHg}$, but it returned to a normal level with medication. The retinal pigment reaction was not clear in 8 eyes, requiring supplementary laser therapy after the operation. 
Table 1 Comparison of variables after anti-CMV induction and the occurrence of retinal detachment between the laser and nonlaser groups

\begin{tabular}{|c|c|c|c|c|c|c|}
\hline Item & Visual acuity & IOP (mmHg) & $\begin{array}{c}\text { Central type/ } \\
\text { peripheral type (eye) }\end{array}$ & $\begin{array}{l}\text { Number of } \mathrm{CD}^{+} \mathrm{T} \\
\text { cells }(\mathrm{N} / \mu \mathrm{L})\end{array}$ & HIV viral load (copy $\mathrm{mL}^{-1}$ ) & $\begin{array}{c}\text { Eyes with retinal } \\
\text { detachment (N/\%) }\end{array}$ \\
\hline Nonlaser group $(n=50)$ & $0.54 \pm 0.32$ & $11[8-18]$ & $19 / 31$ & 14 [2-48] & $105,262[5,812-520,128]$ & $12 / 24 \%$ \\
\hline Laser group $(n=46)$ & $0.56 \pm 0.29$ & $11[6-15]$ & $19 / 27$ & $21[1-48]$ & $69,442[1,256-462,438]$ & $3 / 6.52 \%$ \\
\hline$T / Z / \chi^{2}$ & -0.217 & -0.409 & 0.109 & -1.178 & -1.485 & 5.552 \\
\hline
\end{tabular}

anti-CMV, anticytomegalovirus; IOP, intraocular pressure.

Table 2 The occurrence of retinal detachment in the laser and nonlaser groups

\begin{tabular}{lccc}
\hline Item & Number of eyes $(\mathrm{N})$ & Eyes with retinal detachment $(\mathrm{N})$ & Percentage \\
\hline Laser group & 46 & 3 & 6.52 \\
Nonlaser group & 50 & 12 & 24.00 \\
$\chi^{2}$ & & 5.552 & \\
$\mathrm{P}$ & & 0.018 & \\
\hline
\end{tabular}

\section{Visual acuity, IOP, and human immunodeficiency viral load}

The visual acuity, IOP, and HIV viral load at the end of the induction period were compared between the two groups, and the details are presented in Table 1 . The results indicated that there was no statistical significance between the two groups. Among the 96 eyes from the 80 patients treated with anti-CMV therapy in our hospital, 15 had retinal detachment complications. Among the 46 eyes in the laser group, 3 eyes had retinal detachment (an incidence rate of $6.5 \%$ ), while among the 50 eyes in the nonlaser group, retinal detachment occurred in 12 (an incidence rate of $24 \%$ ). Clearly, the incidence of retinal detachment in the nonlaser group was significantly higher than that in the laser treatment group, and the difference was statistically significant $(\mathrm{P}=0.018)$, as shown in Table 2. However, the differences in the number of $\mathrm{CD}^{+}{ }^{+} \mathrm{T}$ cells, HIV viral load, and the time from retinal detachment to the end of the induction period were not statistically significant between the two groups, as shown in Tables 1,2. A typical case is illustrated in Figure 3.

\section{Discussion}

CMVR is the most serious opportunistic eye infection in patients with AIDS, with retinal detachment being one of the main causes of vision loss in patients suffering from the infection. In our hospital, anti-CMV therapy involving an intravenous drip and intravitreal injection of ganciclovir and/or sodium phosphate during the induction period, along the oral administration of ganciclovir during the maintenance period, has achieved good results, generally improving visual quality. However, retinal detachment continues to be a risk for patients. A total of 96 eyes from among 80 AIDS/CMVR patients were treated with antiCMV therapy in our hospital, and 15 developed retinal detachment. We believe that the causes of this retinal detachment may have been correlated with a number of factors. First, the full-thickness retinal necrosis caused by CMVR may inevitably form numerous holes in the necrotic focus, especially in the junctional region, which is likely to cause retinal detachment. Meanwhile, the vitreous liquefaction would be accelerated due to the vitreoretinal inflammation caused by CMV and the disturbance of the vitreous body by the intravitreal injection, while local vitreous liquefaction may occur due to the formation of a retinal hole. If this occurs, the liquified vitreous body will enter the subretinal cavity through the retinal hole, thus leading to retinal detachment (10-12). Finally, where the $\mathrm{CMV}$ virus cannot be completely removed, the retina may undergo chronic inflammatory stimulation and immune reconstruction, which leads to the vitreous opacification and retinal detachment caused by the fibrous proliferative membrane. 

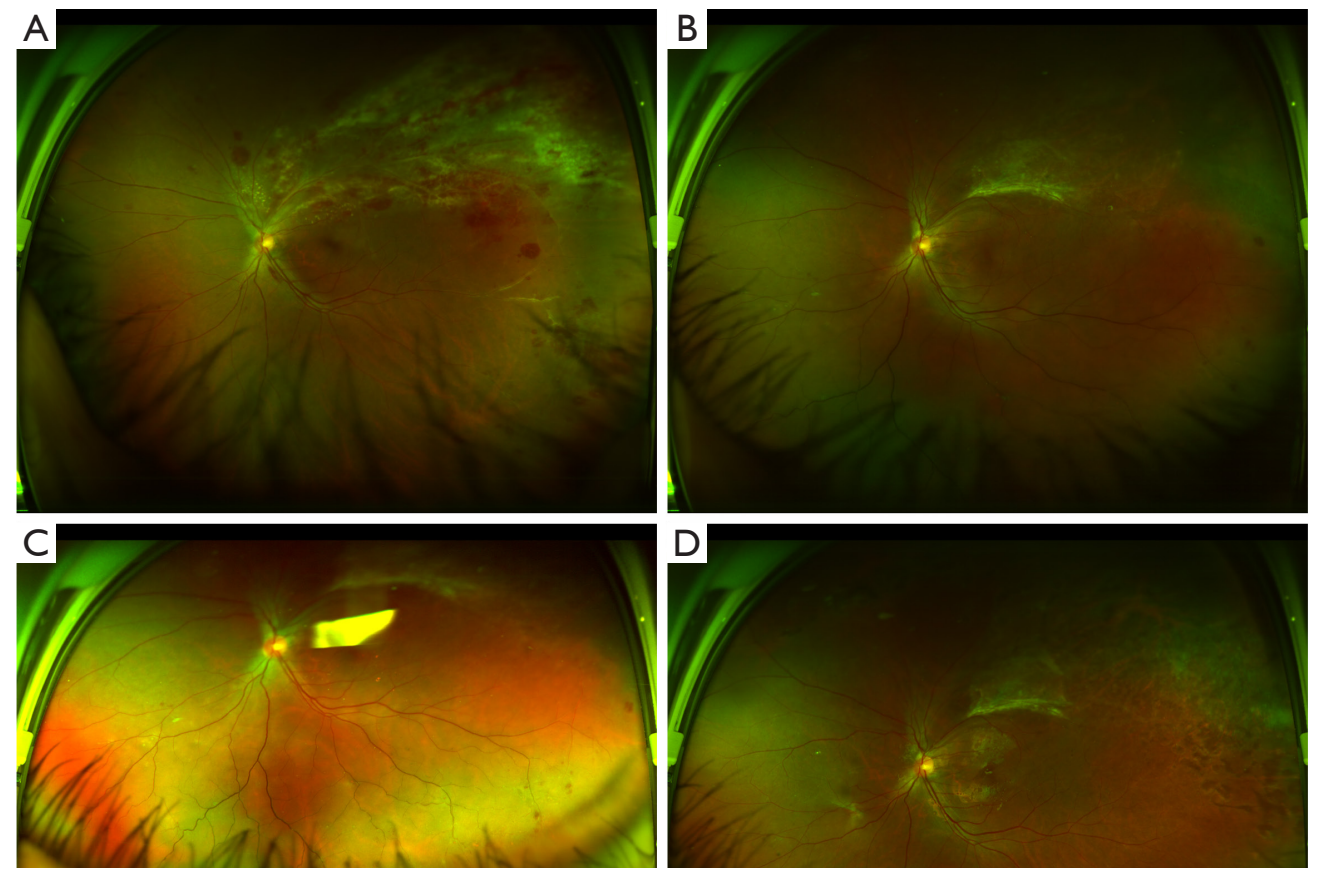

Figure 3 Typical case 3. This case was a nonlaser group patient with retinal detachment. (A) Before anti-CMV induction therapy; (B) after anti-CMV induction therapy; (C) retinal detachment occurring 120 days following commencement of maintenance therapy; D: after vitrectomy. CMVR, cytomegalovirus retinitis; anti-CMV, anticytomegalovirus.

CMVR is a complete retinal necrosis caused by the CMV reaching the retina through the blood flow. Most researchers believe that vascular endothelial cell infection causes the initial CMVR infection, and that this initial infection is more problematic than the spreading of the infection given that few new infection foci surface during the infectious process (13-15). The CMV is mainly diffused via intercellular transmission, which means that the junction between the normal retina and the necrotic retina can be clearly visible in a retina with CMV lesions, making it easier to distinguish via angiography. When the CMVR lesions are under control, most of the junctions remain still and stable. However, through long-term observation and through our comparison of the photos taken by the Oberg camera, it was clear that there were a few cases with unexplained necrosis in the border area, and that the lesions were slowly expanding. We also found that there was no capillary perfusion area in the junction area of a few patients following angiography and blood flow optical coherent tomography examinations. Following the close observation of the changes to the junctional area, and with reference to the recommended methods of laser treatment for preventing retinal detachment in terms of retinal holes and the lattice degeneration of the fundus in high myopia, a 3-4-row encircling intercepting laser photocoagulation was performed on the normal retina of the junction area to prevent retinal detachment and to eliminate the nonperfusion area to stop the disease from progressing. The incidence of retinal detachment was only $6.5 \%$ in the laser group, while that of the nonlaser group was $24 \%$, indicating a statistically significant difference $(\mathrm{P}=0.018)$. However, the differences in the number of $\mathrm{CD}^{+} \mathrm{T}$ cells, the HIV viral load, and the time from retinal detachment to the end of the induction period were not statistically significant between the two groups. Thus, it can be concluded that laser treatment can effectively reduce the incidence of retinal detachment.

In the present study, retinal detachment occurred in 3 eyes following laser therapy. In our experience, all necrotic lesions should be closed via laser treatment. However, some retinal CMVR lesions are comparatively slight, meaning it is difficult to distinguish the junction area with the naked eye, and this area can easily form holes. Therefore, prior to the laser treatment, angiography should be performed to identify the lesion junction area, and all the lesions should subsequently be closed using laser therapy. The 
larger the area of retinal necrosis is in CMVR, the greater the probability of retinal hole formation. Therefore, early control of CMVR results in a smaller area of retinal necrosis. Meanwhile, with any increase in lesion size, a larger part of the normal retinal area must be closed to prevent retinal detachment. An intravitreal injection of ganciclovir can rapidly control the lesions deepening and extending to the peripheral area (16-18). Overall, this indicates that early treatment, early diagnosis, and an early intravitreal injection are crucial.

In the present study, the earliest retinal detachment occurred 30 days after the conclusion of the anti-CMV induction period, while the latest occurred 530 days after conclusion of the induction period. Thus, we believe that when a patient commences the maintenance period, CMVR will generally be stable, the lesion area will likely not expand, and the patient's general condition should be robust enough to withstand the laser treatment, allowing laser therapy to be conducted. On conclusion of the induction therapy in the present study, patients demonstrated a range of $\mathrm{CD}^{+}{ }^{+} \mathrm{T}$ cells of $1-48$ cells $/ \mu \mathrm{L}$, and an $\mathrm{HIV}$ viral load range from 5,812 to 520,128 copies $\mathrm{mL}^{-1}$. Although the patients have received HAART treatment when they received laser treatment, their infectivity is still very strong, and the patients often have tuberculosis, syphilis or other infectious diseases, requiring our hospital to develop a specific laser treatment program for patients with AIDS in order to protect both the doctors and the patients.

Following laser therapy, KP, anterior chamber flare, and elevated IOP often emerge, which may be correlated with CVMR alone. Although there were no serious adverse reactions to the laser therapy in the present study, it is important to ensure that a routine review is conducted, and a timely administration of the appropriate drugs is performed in the case of adverse reactions. We believe that performing prophylactic laser therapy during the maintenance period of CMVR is a safe and feasible option. However, in future work, we will investigate the theoretical basis of individualized laser therapy for patients undergoing the CMVR induction period and will accordingly formulate a feasible protocol.

\section{Conclusions}

Laser therapy can effectively reduce the incidence of retinal detachment after the induction period of anti-CMV treatment in patients with AIDS and CMVR, and the therapy is safe and reliable.

\section{Acknowledgments}

Funding: This work was supported by the National 13th Five-Year Grand Program on Key Infectious Disease Control (No. 2018ZX10301407-005-001 to TYJ, No. 2018ZX10302103-001-003 to TYJ), and the Scientific Research Project of Beijing YouAn Hospital, CCMU, 2018 (No. YNKTQN20180201).

\section{Footnote}

Conflicts of Interest: All authors have completed the ICMJE uniform disclosure form (available at http://dx.doi. org/10.21037/qims-20-990). There are no conflicts of interest to declare.

Ethical Statement: This study was approved by the Capital Medical University Institutional Review Board of Beijing YouAn Hospital (LL-2018-150-K) and adhered to the tenets of the Declaration of Helsinki. Written informed consent was obtained from all participants.

Open Access Statement: This is an Open Access article distributed in accordance with the Creative Commons Attribution-NonCommercial-NoDerivs 4.0 International License (CC BY-NC-ND 4.0), which permits the noncommercial replication and distribution of the article with the strict proviso that no changes or edits are made and the original work is properly cited (including links to both the formal publication through the relevant DOI and the license). See: https://creativecommons.org/licenses/by-nc-nd/4.0/.

\section{References}

1. Sugar EA, Jabs DA, Ahuja A, Thorne JE, Danis RP, Meinert CL; Studies of the Ocular Complications of AIDS Research Group. Incidence of cytomegalovirus retinitis in the era of highly active antiretroviral therapy. Am J Ophthalmol 2012;153:1016-24.e5.

2. Holland GN. AIDS and ophthalmology: the first quarter century. Am J Ophthalmol 2008;145:397-408.

3. Jabs DA, Bartlett JG. AIDS and ophthalmology: a period of transition. Am J Ophthalmol 1997;124:227-33.

4. Schrier RD, Song MK, Smith IL, Karavellas MP, Bartsch DU, Torriani FJ, Garcia CR, Freeman WR. Intraocular viral and immune pathogenesis of immune recovery uveitis in patients with healed cytomegalovirus retinitis. Retina 2006;26:165-9. 
5. Sun HY, Mao FF, Li D, Wang SN, Liu YH, Xu XJ, Liu BB, Dong Y. Clinical characteristics and prognosis of cytomegalovirus retinitis with acquired immune deficiency syndrome without anticytomegalovirus therapy. Ophthalmology in China 2016;25:195-8.

6. Wong JX, Wong EP, Teoh SC. Outcomes of cytomegalovirus retinitis-related retinal detachment surgery in acquired immunodeficiency syndrome patients in an Asian population. BMC Ophthalmol 2014;14:150.

7. Chew GWM, Teoh SCB, Agrawal R. Analysis and outcomes of cataract surgery in patients with acquired immunodeficiency syndrome. Ocul Immunol Inflamm 2017;25:535-9.

8. Ye JJ, Li HY, Sun D, Min HY, Han BL, Hu TS. Cytomegalovirus retinitis associated with acquired immunodeficiency syndrome. Zhonghua Yan Ke Za Zhi 2005;41:803-6.

9. Althaus C, Loeffler KU, Schimkat M, Hudde T, Sundmacher R. Prophylactic argon laser coagulation for rhegmatogenous retinal detachment in AIDS patients with cytomegalovirus retinitis. Graefes Arch Clin Exp Ophthalmol 1998;236:359-64.

10. Davis JL, Hummer J, Feuer WJ. Laser photocoagulation for retinal detachments and retinal tears in cytomegalovirus retinitis. Ophthalmology 1997;104:2053-60; discussion 2060-1.

11. Zhang CF. Fundus epidemiology. 1st ed. Beijing: People's
Medical Publishing House, 1998:371-8.

12. Zhang CF. Fundus epidemiology. 2nd ed. Beijing: People's Medical Publishing House, 2010:408-17.

13. Boss JD, Rosenberg K, Shah R. Dual intravitreal injections with foscarnet and ganciclovir for ganciclovir-resistant recurrent cytomegalovirus retinitis in a congenitally infected infant. J Pediatr Ophthalmol Strabismus 2016;53:e58-60.

14. Yeh S, Forooghian F, Faia LJ, Weichel ED, Wong WT, Sen HN, Chan-Kai BT, Witherspoon SR, Lauer AK, Chew EY, Nussenblatt RB. Fundus autofluorescence changes in cytomegalovirus retinitis. Retina 2010;30:42-50.

15. Tawse KL, Baumal CR. Intravitreal foscarnet for recurring CMV retinitis in a congenitally infected premature infant. J AAPOS 2014;18:78-80.

16. Ausayakhun S, Yuvaves P, Ngamtiphakom S, Prasitsilp J. Treatment of cytomegalovirus retinitis in AIDS patients with intravitreal ganciclovir. J Med Assoc Thai 2005;88 Suppl 9:S15-20.

17. Fan JJ, Tao Y, Hwang DK. Comparison of intravitreal ganciclovir monotherapy and combination with foscarnet as initial therapy for cytomegalovirus retinitis. Int J Ophthalmol 2018;11:1638-42.

18. Miao H, Tao Y, Jiang YR, Li XX. Multiple intravitreal injections of ganciclovir for cytomegalovirus retinitis after stem-cell transplantation. Graefes Arch Clin Exp Ophthalmol 2013;251:1829-33.

Cite this article as: $\mathrm{Xie} \mathrm{LY}, \mathrm{Li} \mathrm{XN}$, Chen C, Kong WJ, Jiang TY, Du KF, Dong HW, Guo CG, Wei WB. Clinical efficacy of laser therapy in the prevention of retinal detachment in patients with acquired immunodeficiency syndrome and cytomegalovirus retinitis. Quant Imaging Med Surg 2021;11(6):2634-2641. doi: 10.21037/qims-20-990 